\title{
Role of ledipasvir/sofosbuvir combination for genotype I hepatitis C virus infection
}

\author{
This article was published in the following Dove Press journal: \\ Hepatic Medicine: Evidence and Research \\ 28 June 2016 \\ Number of times this article has been viewed
}

\author{
Vinay Sundaram' \\ Kris V Kowdley ${ }^{2}$ \\ 'Department of Medicine and \\ Comprehensive Transplant Center, \\ Cedars-Sinai Medical Center, Los \\ Angeles, CA, ${ }^{2}$ Liver Care Network, \\ Swedish Medical Center, Seattle, WA, \\ USA
}

\begin{abstract}
Chronic hepatitis $\mathrm{C}$ virus (HCV) infection is one of the most common etiologies of liver-related mortality throughout the world. Among the six HCV genotypes, genotype 1 was significantly more aggressive when utilizing the combination of pegylated interferon and ribavirin, as genotype 1 -infected patients had the lowest likelihood of achieving cure $(40 \%-50 \%)$ and required twice as long duration of treatment, as compared to genotypes 2 and 3 . Recently, however, significant advances have been made with the advent of all-oral direct-acting antiviral agents, which have significantly improved the safety, efficacy, and tolerability of the treatment of HCV genotype 1. Among the available treatments for HCV genotype 1, the combination therapy of ledipasvir/sofosbuvir provides several advantages compared to other regimens, including use of a single-pill regimen, possibility to shorten the duration of treatment to 8 weeks, efficacy in patients exposed to protease inhibitors, safety in decompensated cirrhosis, and potential to avoid ribavirin. In this review, we discuss the pharmacotherapy of the combination of ledipasvir/ sofosbuvir therapy and summarize the results of the Phase III clinical trials for this treatment in HCV genotype 1 patients. We will also discuss the data for special populations, including decompensated cirrhosis, human immunodeficiency virus (HIV) coinfected patients, AfricanAmericans, the elderly, and those who failed sofosbuvir-containing regimens.
\end{abstract}

Keywords: pegylated interferon, ribavirin, cirrhosis, liver transplantation, direct-acting antiviral

\section{Overview}

Affecting nearly $130-170$ million persons throughout the world, or $\sim 2 \%-3 \%$ of the world's population, hepatitis $\mathrm{C}$ virus (HCV) is the leading cause of liver-related mortality and liver transplantation. ${ }^{1,2}$ If left untreated, chronic HCV infection may progress to cirrhosis with complications of end-stage liver disease or hepatocellular carcinoma. ${ }^{1}$ Furthermore, nonhepatic sequelae may occur due to untreated infection, including cryoglobulinemia, porphyria cutanea tarda, and B-cell lymphoma. ${ }^{3}$ To date, six HCV genotypes have been identified, of which genotype 1 is the most prevalent in the US and Europe, followed by genotype $3 .^{4}$

For the last 15 years, pegylated interferon (PEG-IFN) in combination with ribavirin (RBV) was the primary mode of treatment for $\mathrm{HCV}$, through targeted boosting of the patient's immune system. Clinical trials have demonstrated that HCV genotype 1 had the lowest sustained virologic response (SVR) rate to such therapy (40\%-50\%) compared to genotypes $2(80 \%-90 \%)$ and $3(60 \%-70 \%){ }^{5,6}$ These findings, combined with a significantly greater prevalence of genotype 1 in the Western world, have led to an increased focus of research on new antiviral agents for HCV genotype 1. ${ }^{4}$ In 2011,
Correspondence: Kris V Kowdley Liver Care Network, Swedish Medical Center, I I0I Madison St., Suite 200, Seattle, WA 98104, USA

$\mathrm{Tel}+|206215| 437$

Fax + I 206320743 I

Email Kris.Kowdley@swedish.org 
the first generation of direct-acting antiviral agents (DAAs) against the nonstructural (NS)3/4 protease were added to PEG-IFN and RBV therapy, leading to increased SVR rates of $75 \% .{ }^{6}$ Over the last 2 years, additional DAAs have been developed for HCV genotype 1, which allow for all oral therapy potentially without RBV, incorporate multiple different targets of the HCV replication pathway and yield cure rates $>90 \%{ }^{7}$

Among the second-generation DAAs, sofosbuvir (SOF) (brand name Sovaldi), a nucleotide analog inhibitor of the nonstructural protein $5 \mathrm{~B}$ (NS5B) viral polymerase ${ }^{8}$ is the first to demonstrate activity against all HCV genotypes. ${ }^{9-11}$ Ledipasvir (LDV) is a nonstructural protein 5A (NS5A) replication complex inhibitor that has been approved in the US since October 2014 for treatment of HCV in combination with SOF, as a fixed-dose single pill called Harvoni. The recommendation for treatment-naive patients with genotype 1 infection without cirrhosis is the fixed-dose combination of LDV/SOF for 12 weeks without RBV. ${ }^{12}$ However, patients with viral load $<6$ million $\mathrm{IU} / \mathrm{mL}$ can be considered for 8 weeks' therapy. ${ }^{12}$ However, for patients who are treatmentexperienced without cirrhosis, treatment should be given for 12 weeks, without RBV. Among patients with cirrhosis, 12 weeks of therapy is recommended for the treatmentnaive, while 24 weeks is recommended for those who are treatment-experienced. ${ }^{12,13}$

In this article, we review the combination of LDV/SOF for treatment of HCV genotype 1, with a focus on data from Phase II and III studies, findings from real-world cohorts. We also discuss the safety and efficacy of LDV/SOF treatment for special patient populations.

\section{Mechanism of action}

The HCV genome encodes for ten proteins, of which three structural proteins are located off the N-terminus and seven NS proteins are positioned off the C-terminus. ${ }^{14}$ The three NS proteins, known as NS3/4A, NS5A, and NS5B are necessary for HCV replication, and the medications which target these proteins are known as DAAs. ${ }^{15} \mathrm{NS} 3 / 4 \mathrm{~A}$ is a serine protease critical for viral replication. ${ }^{15} \mathrm{NS} 5 \mathrm{~B}$ is an RNA polymerase that is also necessary for HCV replication. As the catalytic site of NS5B is highly conserved across all genotypes of $\mathrm{HCV}$, nucleotide inhibitors of NS5B, such as SOF, have been associated with pan-genotypic activity, high potency, and high barrier to resistance. ${ }^{15}$ NS5A is a zinc-binding phosphoprotein that comprises three distinct domains. ${ }^{16,17}$ Domains I, II, and III are necessary for viral RNA replication, with domain
III having specific importance in viral particle assembly. ${ }^{18,19}$ LDV shows activity against the NS5A replication complex. ${ }^{20}$

\section{Phase III studies}

The ION-1 study was one of the first Phase III clinical trials to evaluate the efficacy of LDV/SOF in HCV genotype 1 -infected patients who were treatment-naive. ${ }^{21} \mathrm{~A}$ total of 865 patients were enrolled, among which $20 \%$ had cirrhosis, to receive LDV/SOF for 12 or 24 weeks, both with and without RBV. Among the four treatment arms, the SVR12 rates ranged from $97 \%$ to $99 \%$ across all arms. The conclusion from the ION-1 was that 12 weeks of therapy was similarly effective as 24 weeks of treatment and that the addition of ribavirin did not significantly increase SVR 12 rates, even among patients with cirrhosis. ${ }^{21}$

In the ION-2 trial, 440 treatment-experienced $(20 \%$ with cirrhosis), HCV genotype 1-infected patients who had failed PEG-IFN/RBV with or without telaprevir or boceprevir were randomized to receive 12 or 24 weeks of LDV/SOF with or without $\mathrm{RBV}^{22}$ In the total patient population, the SVR12 rates for 12 weeks of LDV/SOF were $94 \%$ without RBV and $96 \%$ with RBV. For 24 weeks of LDV/SOF treatment, the SVR12 rates were $99 \%$ both with and without RBV. Overall, the recommendation was that noncirrhotic patients who had failed prior treatment, including telaprevir or boceprevir, should be treated with LDV/SOF for 12 weeks without RBV. However, the analysis of patients with cirrhosis demonstrated SVR12 rates of $86 \%$ and $82 \%$ for 12 weeks of treatment without and with RBV, respectively. However, SVR12 rates were significantly higher in this group if receiving 24 weeks of LDV/SOF, either with (99\%) or without RBV (99\%). Therefore, among the treatment-experienced patients with cirrhosis, the investigators concluded that 24 weeks of treatment is needed, though the addition of RBV does not appear to improve efficacy. ${ }^{22}$

In the ION-3 trial, 647 treatment-naive, HCV genotype 1 -infected patients without cirrhosis were studied, to assess whether the treatment duration of LDV/SOF could be shortened to 8 weeks, either with or without RBV. ${ }^{23}$ This was a noninferiority study that showed similar SVR rates between the regimens, with an SVR12 of $94 \%$ with 8 weeks of LDV/ SOF, 93\% with 8 weeks of LDV/SOF with RBV, and 95\% with 12 weeks of LDV/SOF. However, a greater percentage of patients treated for 8 weeks experienced virologic relapse after stopping therapy (4.6\%) compared to those treated for 12 weeks (2\%). ${ }^{23}$ Post hoc analyses revealed that patients with baseline HCV RNA level $<6$ million $\mathrm{IU} / \mathrm{mL}$ who received 8 weeks of therapy had a similar relapse rate, as those who 
received 12 weeks of therapy, suggesting that 8 weeks of treatment can be considered in such patients. ${ }^{23}$ Furthermore, RBV provided no additional benefits with regard to increasing SVR rates and therefore is not necessary in patients receiving 8 weeks of therapy. ${ }^{23}$

\section{Decompensated cirrhosis}

In the SOLAR-1 trial, the efficacy and safety of LDV/SOF with RBV for 12 or 24 weeks were evaluated in 108 patients with decompensated cirrhosis. ${ }^{24}$ The study included ChildTurcotte-Pugh (CTP) class B or C cirrhosis and the majority of patients had a baseline model for end-stage liver disease scores between 10 and 20 . SVR12 rates were $87 \%$ for 12 weeks of treatment and $89 \%$ for 24 weeks of treatment, and no differences in SVR rates were found between patients with CTP class B or C cirrhosis. ${ }^{24}$ Additionally, antiviral therapy was associated with improved model for end-stage liver disease scores in most patients $(60 \%-79 \%)$. A total of three patients discontinued therapy as a result of adverse events (AEs). ${ }^{24}$

The SOLAR-2 study similarly explored patients with decompensated cirrhosis, including CTP class B or C. ${ }^{25}$ Patients were randomly assigned to receive LDF/SOF with RBV for 12 or 24 weeks. A total of 333 patients were enrolled, of which 296 were HCV genotype 1. Among patients with genotype $1 \mathrm{HCV}$, SVR12 was achieved by $20 / 23$ (87\%) of CTP-B patients receiving 12 weeks of treatment, 22/23 $(96 \%)$ of patients with 24 weeks of treatment, 17/20 (85\%) of CTP-C patients receiving 12 weeks, and 18/23 (78\%) of CTP-C patients undergoing 24 weeks of treatment. ${ }^{25} \mathrm{~A}$ total of seven patients ( $2 \%$ ) stopped treatment prematurely secondary to AEs, and 17 patients died secondary to complications of hepatic decompensation. ${ }^{25}$

In the SIRUS trial, the efficacy of a 12-week course of LDV/SOF plus RBV ( $n=77)$ was compared to a 24-week course of LDV/SOF $(n=78)$, in treatment-experienced patients with $\mathrm{HCV}$ genotype 1 infection and compensated cirrhosis who failed prior treatment with IFN-based therapy, which also utilized telaprevir or boceprevir. ${ }^{26}$ The SVR12 rates were $96 \%$ for patients in the LDV/SOF plus RBV group receiving 12 weeks of therapy and $97 \%$ in the LDF/ SOF group treated for 24 weeks. ${ }^{26}$ This study suggests that in genotype 1 treatment-experienced patients with cirrhosis, a 12-week course of LDV/SOF plus RBV may provide a more cost-effective regimen with SVR12 rates similar to those receiving 24 weeks of $\mathrm{LDV} / \mathrm{SOF}$ without $\mathrm{RBV}$.

Additionally, a pooled analysis was performed by Reddy et al of 513 patients with compensated cirrhosis from Phase
II and III clinical trials, of which $70 \%$ were treatmentexperienced. ${ }^{27}$ The overall efficacy of treatment with LDV/ SOF in patients with cirrhosis was $96 \%$, specifically $98 \%$ among treatment-naive and 95\% among treatment-experienced individuals. The lowest rate of SVR12 occurred in treatment-experienced patients who received 12 weeks of therapy without RBV (90\%). ${ }^{27}$ However, among this cohort, adding RBV and extending the treatment duration from 12 to 24 weeks improved the likelihood of reaching SVR. The presence of portal hypertension and severity of liver disease were additional predictors for reduced SVR rates. ${ }^{27}$ Based on this analysis, $\mathrm{HCV}$ guidance recommended that treatment-naive patients with cirrhosis be treated with 12 weeks of LDV/SOF alone, and treatment-experienced patients with cirrhosis be treated with 24 weeks of LDV/SOF alone or with 12 weeks of LDV/SOF plus RBV. ${ }^{12}$

\section{Relapsers to SOF-based treatment}

In an open-label trial, Wyles et al studied the efficacy of LDV/ SOF plus RBV for 12 weeks in HCV genotype 1-infected patients who failed previous SOF-based regimens. ${ }^{28}$ Among the 51 patients enrolled, 25 had previously received SOF plus PEG-IFN/RBV, 20 had received SOF and RBV, five had received SOF placebo plus PEG-IFN alfa and RBV, and one had received monotherapy with GS-0938, an NS5B polymerase inhibitor. SVR12 was achieved by 50 of the 51 patients $(98 \%)$ treated. ${ }^{28}$ This study therefore demonstrated that SOF-based therapy can be used to re-treat patients who were previously exposed to SOF, since there is low likelihood of the virus developing a SOF resistant mutation. Furthermore, SOF-resistant HCV variants, such as the S282T mutation, are markedly less fit for viral replication than wildtype variants, which may explain why retreatment with SOF in previously exposed individuals is successful. ${ }^{13}$

In contrast, patients who failed regimens that included an NS5A inhibitor, including LDV/SOF, are likely to have resistance-associated variants to NS5A inhibitors, which can persist for years and reduce the efficacy with NS5A inhibitor therapy. ${ }^{29}$ In one study, of the 41 patients who previously failed LDV/SOF therapy for 8 or 12 weeks, $71 \%$ achieved SVR12 after retreatment with LDV and SOF for 24 weeks. ${ }^{29}$ SVR12 rates were lower in patients with baseline NS5A resistance-associated variants. Additionally, four patients with NS5A resistance-associated variants developed evidence of SOF resistance. ${ }^{29}$ These findings indicate that NS5A inhibitors, such as LDV, should not be reused in patients who harbor a resistant variant after prior treatment with these agents. 


\section{Special populations}

\section{HIV coinfection}

In the ION-4 study, HCV genotype 1 and four patients, coinfected with HIV received LDV/SOF for 12 weeks, with the primary outcome of SVR $12 .{ }^{30}$ Of the 335 total patients, $55 \%$ were treatment-experienced and $20 \%$ had cirrhosis. The rates of SVR 12 were $96 \%$ in patients with HCV genotype 1a and $96 \%$ in those with HCV genotype $1 b .^{30}$ Subgroup analysis revealed SVR12 rates to be similar regardless of previous treatment or the presence of cirrhosis. Of the 13 patients who did not have an SVR, two had on-treatment failure, while ten had a relapse after the end of treatment. No patient had confirmed HIV virologic breakthrough or rebound. Common AEs, including headache (25\%), fatigue (21\%), and diarrhea (11\%), were similar, regardless of the HIV regimen given. ${ }^{30}$

\section{Elderly patients}

A recent review by Saab et al of data from the ION studies and the GS-US-337-0113 performed in Japan, evaluated the safety and efficacy of LDV/SOF among patients over the age of $65 .{ }^{31}$ Of the 2,293 subjects enrolled in four Phase III trials, 264 (12\%) were $\geq 65$ years of age, of whom 24 were aged $\geq 75$ years. Sustained virological response at 12 weeks was achieved by $97 \%(1,965 / 2,029)$ of subjects aged $<65$ years and $98 \%(258 / 264)$ of subjects aged $\geq 65$ years. Both AEs and rates of study discontinuation were similar between elderly and nonelderly individuals. The use of RBV in 1,042 (45\%) subjects increased the number of AEs, without significantly improving SVR12 rates. $^{31}$

\section{African-Americans}

African-Americans were traditionally viewed as difficult to treat with IFN-based therapy, due to lower response rates often deemed secondary to having an unfavorable interleukin 28B polymorphism. However, a post hoc analysis of the ION studies revealed that among the 308 African-American patients enrolled, 95\% had an SVR12 when taking LDV/SOF, as compared to $97 \%$ of nonblack patients..$^{32}$ No observed differences in SVR rates were found with regards to body mass index, patient age, or presence of interleukin 28B non-CC genotype. Furthermore, the addition of RBV did not affect SVR12 rates in the African-American population. ${ }^{32}$

\section{Posttransplantation}

In addition to studying patients with decompensated cirrhosis, the SOLAR-2 study also evaluated the efficacy of LDF/ SOF treatment for 12 or 24 weeks with RBV, in HCV-infected patients after liver transplantation. Patients enrolled after transplantation included those without cirrhosis, those with cirrhosis (CTP class A-C), and those with fibrosing cholestatic hepatitis C. ${ }^{25}$ Among patients with genotype $1 \mathrm{HCV}$, SVR12 was achieved by 42/45 (93\%) patients without cirrhosis receiving 12 weeks of treatment and 44/44 (100\%) patients without cirrhosis receiving 24 weeks of treatment. Among patients with cirrhosis, 30/30 (100\%) CTP-A patients had SVR12 with 12 weeks of treatment, whereas $27 / 28$ (96\%) CTP-A patients had SVR12 with 24 weeks of treatment. Among decompensated patients, 19/20 (95\%) CTP-B patients had cure with 12 weeks of treatment, 20/20 (100\%) had SVR12 with 24 weeks of treatment, one of two (50\%) CTP-C patients had SVR12 with 12 weeks of treatment; and 4/5 (80\%) CTP-C patients had cure with 24 weeks of treatment. All five patients with fibrosing cholestatic hepatitis $\mathrm{C}$ achieved SVR12. ${ }^{25}$

\section{Safety and tolerability}

LDV/SOF has been reported to be safe and well tolerated. Data regarding AEs comes primarily from the ION studies. The results from the ION trials confirmed the safety and tolerability of the LDV/SOF regimen both with and without $\mathrm{RBV}^{33}$ Less than $1 \%$ of patients either receiving RBV or not discontinued treatment because of an AE. The most common side effects included headache, insomnia, irritability, rash, and fatigue. Treatment-related AE s occurred in $71 \%$ and $45 \%$ of patients treated with and without RBV, respectively. No patient deaths were reported and none of the study patients developed a hemoglobin level $<9 \mathrm{~g} / \mathrm{dL}$ during treatment. ${ }^{33}$ Details regarding the most commonly seen AEs with the use of LSV/SOF, as determined by an integrated safety analysis of the Phase III ION studies, are depicted in Table 1.

Table I Adverse event profile of LDV/SOF

\begin{tabular}{|c|c|c|c|}
\hline Adverse event, n (\%) & $\begin{array}{l}8 \text { weeks } \\
(n=215)\end{array}$ & $\begin{array}{l}12 \text { weeks } \\
(n=539)\end{array}$ & $\begin{array}{l}24 \text { weeks } \\
(n=326)\end{array}$ \\
\hline Fatigue & $45(2 I)$ & $116(22)$ & $79(24)$ \\
\hline Headache & $30(14)$ & $113(2 I)$ & $79(24)$ \\
\hline Nausea & $15(7)$ & $61(11)$ & $36(11)$ \\
\hline Insomnia & II (5) & $41(8)$ & 30 (9) \\
\hline Diarrhea & $15(7)$ & $40(7)$ & $33(10)$ \\
\hline Irritability & $3(1)$ & $22(4)$ & $21(6)$ \\
\hline Rash & $3(1)$ & $23(4)$ & $21(6)$ \\
\hline Arthralgia & $9(4)$ & $32(6)$ & $27(8)$ \\
\hline Cough & $3(1)$ & $18(3)$ & $21(6)$ \\
\hline Pruritus & $2(1)$ & $21(4)$ & $10(3)$ \\
\hline
\end{tabular}

Note: Alqahtani SA, Afdhal N, Zeuzem S, et al. Safety and tolerability of ledipasvir/ sofosbuvir with and without ribavirin in patients with chronic hepatitis $C$ virus genotype I infection: Analysis of phase III ION trials. Hepatology. 2015;62:25-30.33 With permission from John Wiley and Sons, copyright @2015.

Abbreviation: LDV/SOF, ledipasvir/sofosbuvir. 


\section{Conclusion}

Chronic HCV treatment has evolved dramatically over the last 5 years, with the advent of DAAs that directly target $\mathrm{HCV}$ replication, and the transition from IFN-based therapy to non-IFN-based all-oral treatment. HCV genotype 1, which was traditionally the most difficult to treat during the IFN era, can now be cured with all-oral therapy in as little as 8 weeks. There are several advantages to utilizing LDV/SOF therapy. First, this combination can be given for 8 weeks in certain patients as opposed to 12 weeks' duration with other DAA-based therapy; shorter duration of therapy may not only be desirable for the patient but can also lead to reduced costs. Second, RBV has a potentially unfavorable side effect profile, which includes hemolytic anemia, rash, and teratogenicity. Finally, this regimen has proven safety and efficacy in otherwise difficult-to-treat patients, including those with decompensated cirrhosis and patients who have failed treatment using a protease inhibitor. There are still several unmet needs, primarily demonstration of safety in patients with renal failure. The goal of future therapy will be a regimen that is pan-genotypic and has near $100 \%$ efficacy in all patient groups.

\section{Additional reading}

Additional reading for the practitioner includes practice guidelines published by the American Association for the Study of Liver Diseases/Infectious Disease Society of America. ${ }^{12}$ The website (www.hcvguidelines.org) for their recommendations is updated every 3 months to keep the clinician current with evolving data. Recommendations are centered on medications approved in the US.

Hepatitis C practice guidelines are also provided by European Association for Study of the Liver (EASL) ${ }^{34}$ to guide the practitioner in Europe, as the regimens available in Europe may be different from those approved in the US.

\section{Author contributions}

VS and KVK both contributed equally to conceptualization and study design, including literature search, and drafting and critical revision of the manuscript. Both authors are guarantors. All authors contributed toward data analysis, drafting and revising the paper and agree to be accountable for all aspects of the work.

\section{Disclosure}

VS: Speaker's bureau: Salix, Gilead, Abbvie, BMS, Intercept. Advisory board: Gilead, Abbvie, Janssen, BMS, Intercept. KVK: Grants/Research: Evidera, Gilead, Immuron, Intercept,
Tobira. Advisory board: Abbvie, Achillion, BMS, Evidera, Gilead, Merck, Novartis, Trio Health. Consultant: Abbvie, Gilead. The authors report no other conflicts of interest in this work.

\section{References}

1. Alter MJ, Kruszon-Moran D, Nainan OV, et al. The prevalence of hepatitis C virus infection in the United States, 1988 through 1994. N Engl J Med. 1999;341:556-562.

2. Global Burden of Hepatitis CWG. Global burden of disease (GBD) for hepatitis C. J Clin Pharmacol. 2004;44:20-29.

3. Hu KQ, Tong MJ. The long-term outcomes of patients with compensated hepatitis $\mathrm{C}$ virus-related cirrhosis and history of parenteral exposure in the United States. Hepatology. 1999;29:1311-1316.

4. Cornberg M, Razavi HA, Alberti A, et al. A systematic review of hepatitis $\mathrm{C}$ virus epidemiology in Europe, Canada and Israel. Liver Int. 2011; 31(Suppl 2):30-60.

5. Fried MW, Shiffman ML, Reddy KR, et al. Peginterferon alfa-2a plus ribavirin for chronic hepatitis $\mathrm{C}$ virus infection. $N$ Engl J Med. 2002;347:975-982.

6. Ghany MG, Nelson DR, Strader DB, Thomas DL, Seeff LB; American Association for Study of Liver D. An update on treatment of genotype 1 chronic hepatitis $\mathrm{C}$ virus infection: 2011 practice guideline by the American Association for the Study of Liver Diseases. Hepatology. 2011;54:1433-1444.

7. Sadler MD, Lee SS. Revolution in hepatitis C antiviral therapy. Br Med Bull. 2015;113:31-44.

8. Herbst DA Jr, Reddy KR. Sofosbuvir, a nucleotide polymerase inhibitor, for the treatment of chronic hepatitis C virus infection. Expert Opin Investig Drugs. 2013;22:527-536.

9. Jacobson IM, Gordon SC, Kowdley KV, et al. Sofosbuvir for hepatitis C genotype 2 or 3 in patients without treatment options. $N$ Engl J Med. 2013;368:1867-1877.

10. Lawitz E, Gane EJ. Sofosbuvir for previously untreated chronic hepatitis C infection. N Engl J Med. 2013;369:678-679.

11. Zeuzem S, Dusheiko GM, Salupere R, et al. Sofosbuvir and ribavirin in HCV genotypes 2 and 3. N Engl J Med. 2014;370:1993-2001.

12. AASLD/IDSA/IAS-USA. Recommendations for testing, managing, and treating hepatitis C. 2014. [Updated February 24, 2016]. Available from: http://hcvguidelines.org. Accessed March 19, 2016.

13. Alqahtani S, Sulkowski M. Current and evolving treatments of genotype 1 hepatitis C virus. Gastroenterol Clin North Am 2015;44:825-843.

14. Herbst DA, Reddy KR. NS5A inhibitor, daclatasvir, for the treatment of chronic hepatitis C virus infection. Expert Opin Investig Drugs. 2013;22: 1337-1346

15. Gu M, Rice CM. Structures of hepatitis $\mathrm{C}$ virus nonstructural proteins required for replicase assembly and function. Curr Opin Virol. 2013;3: $129-136$.

16. Tanji Y, Kaneko T, Satoh S, Shimotohno K. Phosphorylation of hepatitis C virus-encoded nonstructural protein NS5A. JVirol. 1995;69:3980-3986.

17. Tellinghuisen TL, Marcotrigiano J, Gorbalenya AE, Rice CM. The NS5A protein of hepatitis C virus is a zinc metalloprotein. J Biol Chem. 2004;279:48576-48587.

18. Appel N, Zayas M, Miller S, et al. Essential role of domain III of nonstructural protein $5 \mathrm{~A}$ for hepatitis $\mathrm{C}$ virus infectious particle assembly. PLoS Pathog. 2008;4:e1000035.

19. Tellinghuisen TL, Foss KL, Treadaway JC, Rice CM. Identification of residues required for RNA replication in domains II and III of the hepatitis C virus NS5A protein. J Virol. 2008;82:1073-1083.

20. Bunchorntavakul C, Reddy KR. Review article: the efficacy and safety of daclatasvir in the treatment of chronic hepatitis $\mathrm{C}$ virus infection Aliment Pharmacol Ther. 2015;42:258-272.

21. Afdhal N, Zeuzem S, Kwo P, et al. Ledipasvir and sofosbuvir for untreated HCV genotype 1 infection. N Engl J Med. 2014;370:1889-1898. 
22. Afdhal N, Reddy KR, Nelson DR, et al. Ledipasvir and sofosbuvir for previously treated HCV genotype 1 infection. N Engl J Med. 2014; 370:1483-1493.

23. Kowdley KV, Gordon SC, Reddy KR, et al. Ledipasvir and sofosbuvir for 8 or 12 weeks for chronic HCV without cirrhosis. $N$ Engl J Med. 2014;370:1879-1888.

24. Charlton M, Everson GT, Flamm SL, et al. Ledipasvir and sofosbuvir plus ribavirin for treatment of $\mathrm{HCV}$ infection in patients with advanced liver disease. Gastroenterology. 2015;149:649-659.

25. Manns M, Samuel D, Gane EJ, et al. Ledipasvir and sofosbuvir plus ribavirin in patients with genotype 1 or 4 hepatitis $C$ virus infection and advanced liver disease: a multicentre, open-label, randomised, phase 2 trial. Lancet Infect Dis. 2016 Epub Feb 18.

26. Bourliere M, Bronowicki JP, de Ledinghen V, et al. Ledipasvir-sofosbuvir with or without ribavirin to treat patients with HCV genotype 1 infection and cirrhosis non-responsive to previous protease-inhibitor therapy: a randomised, double-blind, phase 2 trial (SIRIUS). Lancet Infect Dis. 2015;15(4):397-404.

27. Reddy KR, Bourliere M, Sulkowski M, et al. Ledipasvir and sofosbuvir in patients with genotype 1 hepatitis $\mathrm{C}$ virus infection and compensated cirrhosis: An integrated safety and efficacy analysis. Hepatology. 2015;62(1):79-86.
28. Wyles D, Pockros P, Morelli G, et al. Ledipasvir-sofosbuvir plus ribavirin for patients with genotype 1 hepatitis $C$ virus previously treated in clinical trials of sofosbuvir regimens. Hepatology. 2015;61(6):1793-1797.

29. Lawitz E, Flamm S, Yang JC, et al. Retreatment of patients who failed 8 or 12 weeks of ledipasvir/sofosbuvir-based regimens with ledipasvir/ sofosbuvir for 24 weeks. Presented at: Program and abstracts of the 50th Annual Meeting of the European Association for the Study of the Liver; Vienna, Austria; April 22-26; 2015. Abstract O005.

30. Naggie S, Cooper C, Saag M, et al. Ledipasvir and sofosbuvir for HCV in patients coinfected with HIV-1. N Engl J Med. 2015;373:705-713.

31. Saab S, Park SH, Mizokami M, et al. Safety and efficacy of ledipasvir/ sofosbuvir for the treatment of genotype 1 hepatitis $\mathrm{C}$ in subjects aged 65 years or older. Hepatology. 2016;63(4):1112-1119.

32. Wilder JM, Jeffers LJ, Ravendhran N, et al. Safety and efficacy of ledipasvir-sofosbuvir in black patients with hepatitis $\mathrm{C}$ virus infection: $\mathrm{A}$ retrospective analysis of phase 3 data. Hepatology. 2016;63(2):437-444.

33. Alqahtani SA, Afdhal N, Zeuzem S, et al. Safety and tolerability of ledipasvir/sofosbuvir with and without ribavirin in patients with chronic hepatitis C virus genotype 1 infection: Analysis of phase III ION trials. Hepatology. 2015;62:25-30.

34. (EASL) TEAftSotL. Recommendations on treatment of hepatitis $\mathrm{C}$ 2015. J Hepatol. 2015;63(1):199-236.
Hepatic Medicine: Evidence and Research

\section{Publish your work in this journal}

Hepatic Medicine: Evidence and Research is an international, peerreviewed, open access journal covering all aspects of adult and pediatric hepatology in the clinic and laboratory including the following topics: Pathology, pathophysiology of hepatic disease; Investigation and treatment of hepatic disease; Pharmacology of drugs used for the treatment of hepatic disease. Issues of patient safety and quality of care will also be considered. The manuscript management system is completely online and includes a very quick and fair peer-review system, which is all easy to use. Visit http://www.dovepress.com/testimonials.php to read real quotes from published authors. 